\title{
Essential role of the unfolded protein response regulator GRP78/BiP in protection from neuronal apoptosis
}

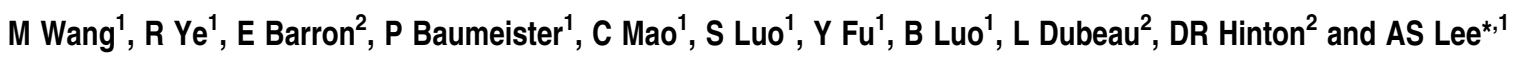

Neurodegenerative diseases are often associated with dysfunction in protein quality control. The endoplasmic reticulum (ER), a key site for protein synthesis, senses stressful conditions by activating the unfolded protein response (UPR). In this study we report the creation of a novel mouse model in which GRP78/BiP, a major ER chaperone and master regulator of UPR, is specifically eliminated in Purkinje cells (PCs). GRP78-depleted PCs activate UPR including the induction of GRP94, PDI, CHOP and GADD34, feedback suppression of elF2 $\alpha$ phosphorylation and apoptotic cell death. In contrast to current models of protein misfolding in which an abnormal accumulation of ubiquitinated protein is prominent, cytosolic ubiquitin staining is dramatically reduced in GRP78-null PCs. Ultrastructural evaluation reveals that the ER shows prominent dilatation with focal accumulation of electron-dense material within the ER. The mice show retarded growth and severe motor coordination defect by week 5 and cerebellar atrophy by week 13. Our studies uncover a novel link between GRP78 depletion and reduction in cytosolic ubiquitination and establish a novel mouse model of accelerated cerebellar degeneration with basic and clinical applications. Cell Death and Differentiation (2010) 17, 488-498; doi:10.1038/cdd.2009.144; published online 9 October 2009

Neurodegenerative diseases are often associated with the formation of toxic intracellular protein aggregates; therefore, it is crucial to understand the factors that regulate their formation and degradation. ${ }^{1}$ The unfolded protein response (UPR) is an evolutionarily conserved mechanism to allow cells to adapt to stress targeted to the endoplasmic reticulum $(E R)^{2}$ Thus, when the protein load exceeds the proteinfolding capacity of the ER, UPR signaling pathways are induced. ER protein quality control is critical for cerebellar Purkinje cell (PC) survival ${ }^{3-5}$ and is linked to the MarinescoSjögren syndrome in humans. ${ }^{6,7}$ ER chaperone, GRP78, also referred to as $\mathrm{BiP} / \mathrm{HSPA} 5$, is a central regulator of $\mathrm{ER}$ function and UPR. ${ }^{2,8,9}$ Tissue culture studies revealed that on ER stress, GRP78/BiP is released from ER transmembrane sensors (PERK, ATF6 and IRE1) resulting in the triggering of the UPR. ${ }^{10}$ However, the direct role of GRP78 in regulating the UPR in vivo and in neurodegeneration is unknown, in part because of the lethality of Grp78-/- embryos. ${ }^{11} \mathrm{PCs}$ are the principal neurons and the sole output of the computational circuitry of the cerebellar cortex, providing signals required for balance, motor coordination and cognitive learning. ${ }^{12}$ The recent discovery that mutations in SIL1, which encodes a nucleotide exchange factor for GRP78, cause progressive multisystem disorder, including cerebellar ataxia in humans ${ }^{6,7}$ and protein accumulation and PC death in SIL1-deficient woozy mutant mice ${ }^{3}$ provides the first hint that ER protein quality control in terminally differentiated neurons is critical for their survival. Interestingly, in woozy mutant mice, GRP78 is upregulated in $\mathrm{PCs},{ }^{3}$ and other co-chaperones such as GRP170 (also known as ORP150) and P58 ${ }^{\text {IPK }}$ (also known as DNAJc3) may serve as alternative nucleotide exchange factors for GRP78. ${ }^{13-16}$ To test directly the role of GRP78 in the UPR in vivo and in neurodegeneration, as well as the mechanism for its putative protective function, here we created a PC-specific knockout mouse model of GRP78. Our studies reveal that complete elimination of GRP78 in PCs leads to specific modulation of the UPR and reduction in cytosolic ubiquitination. GRP78 conditional knockout mice developed PC degeneration by 4.5 weeks, associating with severe motor coordination defect by week 5 and cerebellar atrophy by week 13 , thereby establishing the requirement of GRP78 for PC survival and normal cerebellar function.

\section{Results}

Accelerated PC degeneration and cerebellar atrophy in Grp78 conditional knockout mice. The Grp78 PC-specific knockout mice (abbreviated below as $\mathrm{F} /-$; pc-Cre) were generated by crossing Grp78 F/- mice ${ }^{17}$ with L7Cre-2

\footnotetext{
${ }^{1}$ Department of Biochemistry and Molecular Biology, USC/Norris Comprehensive Cancer Center, University of Southern California Keck School of Medicine, Los Angeles, CA 90089-9176, USA and ${ }^{2}$ Department of Pathology, USC/Norris Comprehensive Cancer Center, University of Southern California Keck School of Medicine, Los Angeles, CA 90089-9176, USA

${ }^{*}$ Corresponding author: AS Lee, Department of Biochemistry and Molecular Biology, USC/Norris Comprehensive Cancer Center, University of Southern California Keck School of Medicine, 1441 Eastlake Avenue, Los Angeles, CA 90089-9176, USA.

Tel: + 323865 0507; Fax: + 323865 0094; E-mail: amylee@ @cnt.usc.edu

Keywords: GRP78/BiP; conditional knockout mice; Purkinje cell survival; unfolded protein response; neurodegeneration

Abbreviations: ER, endoplasmic reticulum; ERAD, ER-associated protein degradation; PCs, Purkinje cells; UPR, unfolded protein response

Received 14.5.09; revised 14.8.09; accepted 02.9.09; Edited by D Rubinsztein; published online 09.10.09
} 
transgenic mice. ${ }^{18}$ In the Grp78 (-) allele, exons 5, 6 and 7 encoding the ATPase domain and peptide-binding domain essential for the chaperone function of GRP78 are deleted (Figure 1a), and no truncated protein was detected. ${ }^{11}$ The genotypes of mice harboring the various Grp78 alleles and the pc-Cre allele were determined by PCR (Figure 1b), and cohorts of mice with genotypes (1) Grp78 +/+, (2) Grp78 F/F, (3) Grp78 F/F; pc-Cre, (4) Grp78 F/- and (5) Grp78 $\mathrm{F} /-$; pc-Cre were monitored for growth and development.

In agreement with previous studies that Grp78 heterozygosity does not affect mouse development and normal organ function, ${ }^{11,19} \mathrm{~F} /$ - mice are phenotypically normal (data a $\frac{\text { ATPase }}{524}$ Peptide-binding
Grp78 Alleles Wild type (+) Floxed (F) Knockout (-)

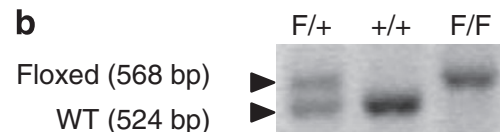

WT (524 bp)

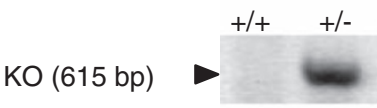

Cre (348 bp) d

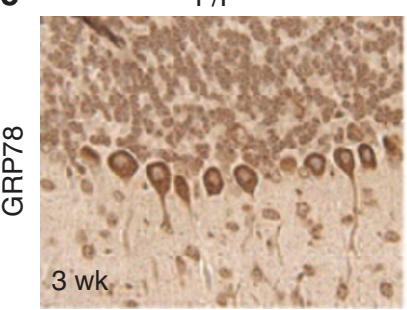

$\mathrm{F} / \mathrm{-}$
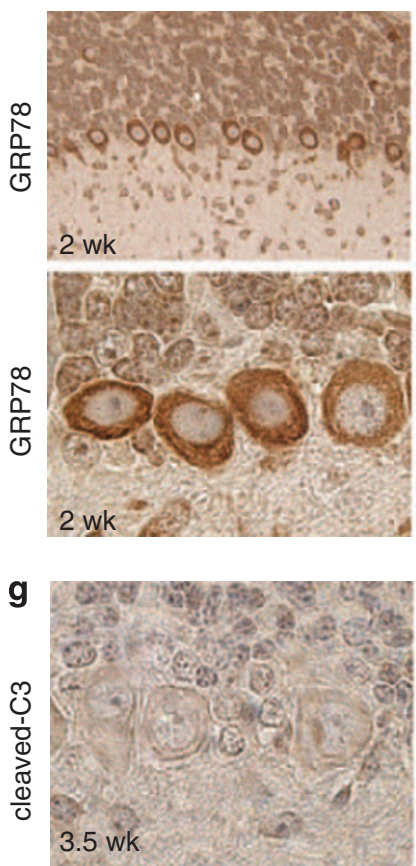

F/-

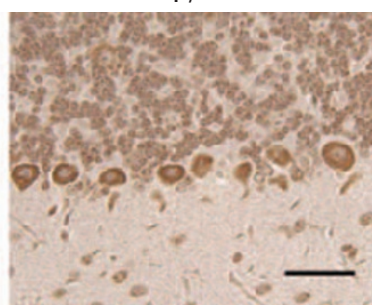

F/-; pc-Cre
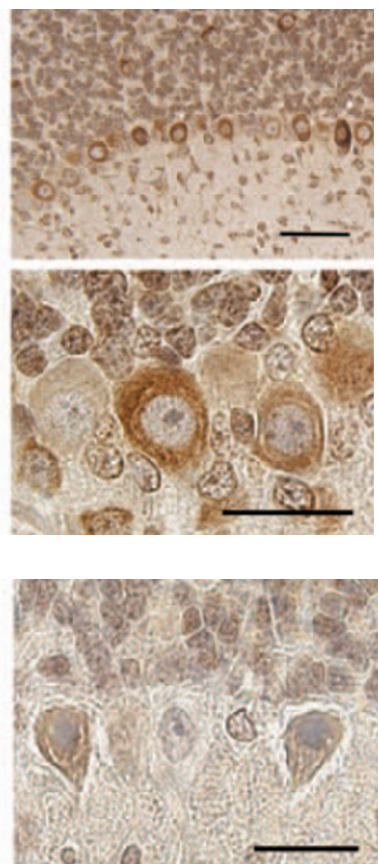

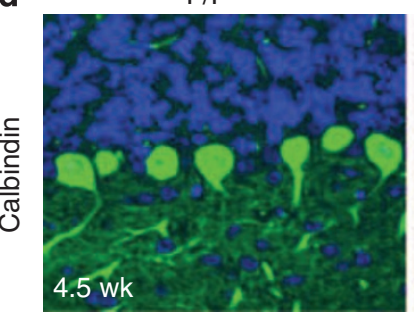

F/-
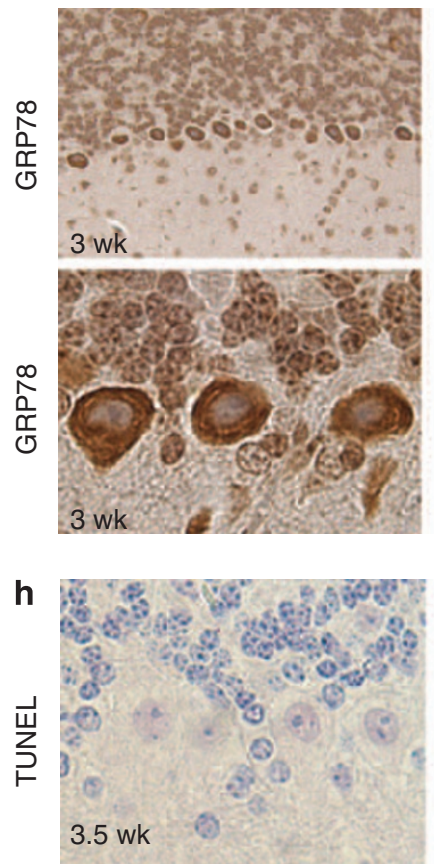

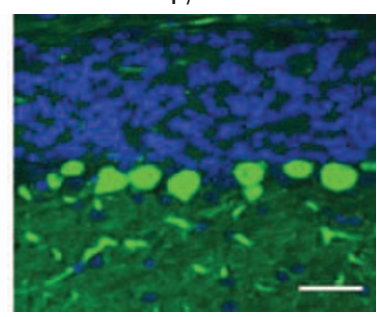

F/-; pc-Cre
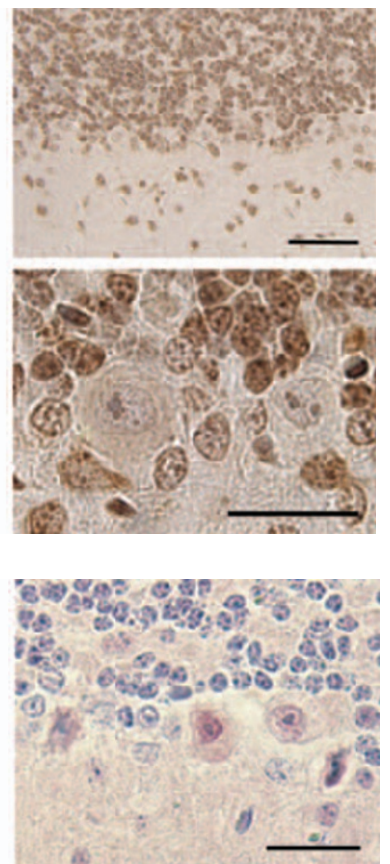

Figure 1 Generation of Grp78 Purkinje cell (PC)-specific knockout mice. (a) Schematic drawing for the Grp78 wild-type (wt) allele (+), floxed allele (F) and knockout (KO) allele (-). Black arrowheads indicate loxP sites. The bars with numbers indicate the size and position of PCR products. (b) Representative genotyping results of Grp78 wt, floxed and KO alleles, and Cre transgene with expected PCR product size. (c) Representative immunohistochemical (IHC) staining for GRP78 shows similar expression levels of GRP78 in the PCs of Grp78F/F mice and their F/- littermates at 3 weeks of age. Staining was performed on sagittal sections of the cerebellum of Grp78 F/F and F/- mice. Scale bar represents $50 \mu \mathrm{m}$. (d) Representative immunofluorescence (IF) staining for calbindin shows there is no PC degeneration in Grp78F/-mice compared with their F/F littermates at 4.5 weeks of age. Green: calbindin; blue: DAPI. Scale bar represents $50 \mu \mathrm{m}$. (e, f) Representative IHC staining for GRP78 on the cerebellum of Grp78 F/- and their F/-; pc-Cre littermates at 2 weeks (e) and 3 weeks (f) of age. Scale bars represent $50 \mu \mathrm{m}$ (upper panel) and $25 \mu \mathrm{m}$ (lower panel). (g, h) Representative IHC staining for cleaved caspase-3 (g) and TUNEL (h) on PCs of Grp78 F/- and their F/-; pc-Cre littermates at 3.5 weeks old. Scale bars represent $25 \mu$ m. For panels c-h, $n=3$ mice per group were examined 
not shown) and their PCs are intact and showed similar GRP78 staining as that of F/F mice (Figure 1c and d). In F/-; pc-Cre mice, Cre recombinase was expressed specifically in PCs after postnatal day 6 , causing a deletion of exon 5 to exon 7 of Grp78. Immunohistochemical analysis revealed that at 2 weeks of age, GRP78 staining was partially reduced in about $10 \%$ of the PCs of F/-; pc-Cre mice compared with that of F/- control, and by week 3, GRP78 was undetectable in about $90 \%$ of PCs (Figure $1 \mathrm{e}$ and $\mathrm{f}$ ). Caspase- 3 activation and positive TUNEL staining were detected in about $10 \%$ of PCs at week 3.5 (Figure $1 \mathrm{~g}$ and $\mathrm{h}$ ), suggesting the onset of PC apoptosis.

Staining for the PC-specific marker calbindin confirmed a partial loss of PCs at week 4.5 (Figure 2a), and by week 13, all 10 lobules of the cerebellum showed extensive PC degeneration (Figure 2b). Correlating with PC loss, cerebellar atrophy was evident in F/-; pc-Cre mice by week 13 (Figure 2c). Quantification of the age-dependent PC loss is summarized in Figure 2d. Collectively, these results establish that GRP78 is required for $\mathrm{PC}$ survival and cerebellum integrity.

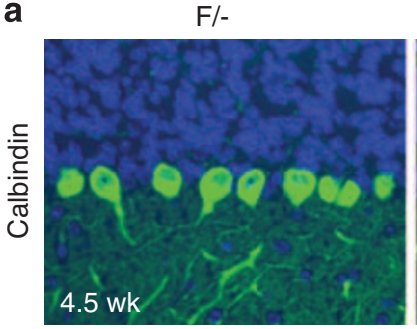

F/-; pc-Cre

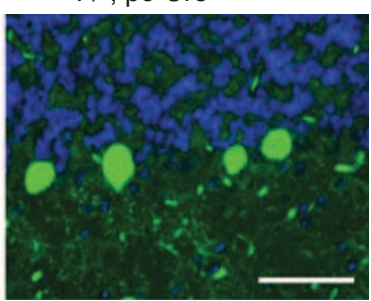

$\mathrm{F} / \mathrm{-}$

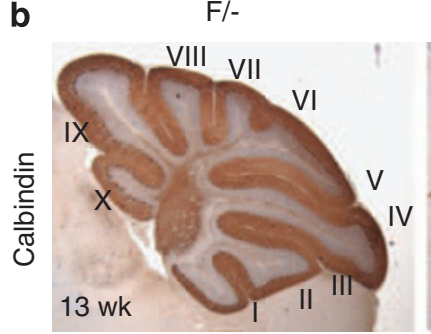

F/-; pc-Cre

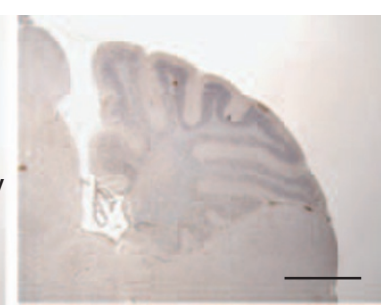

C

F/-
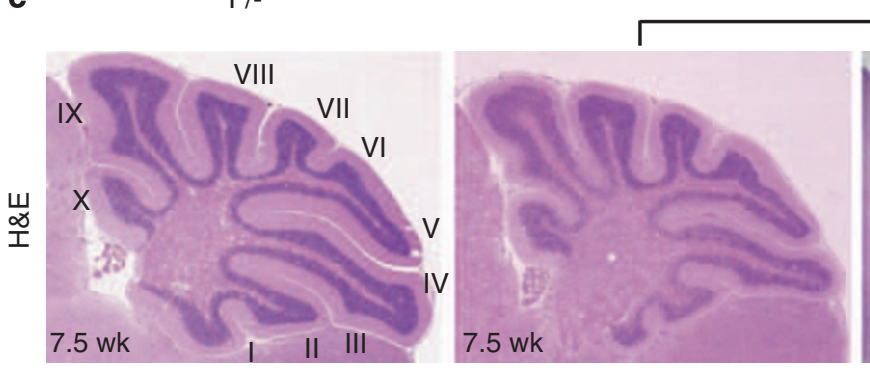

F/-; pc-Cre
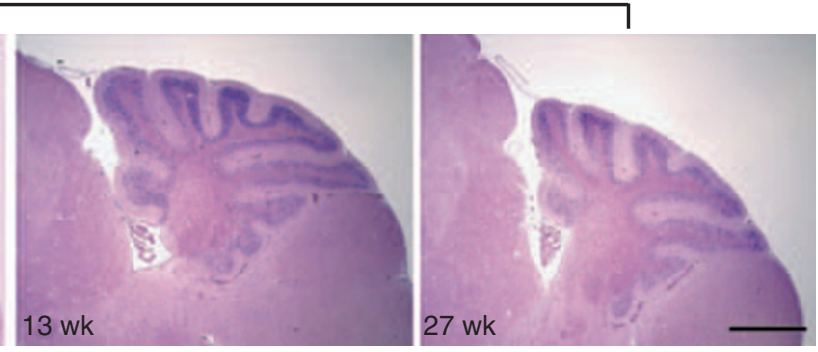

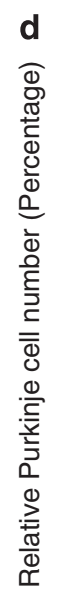

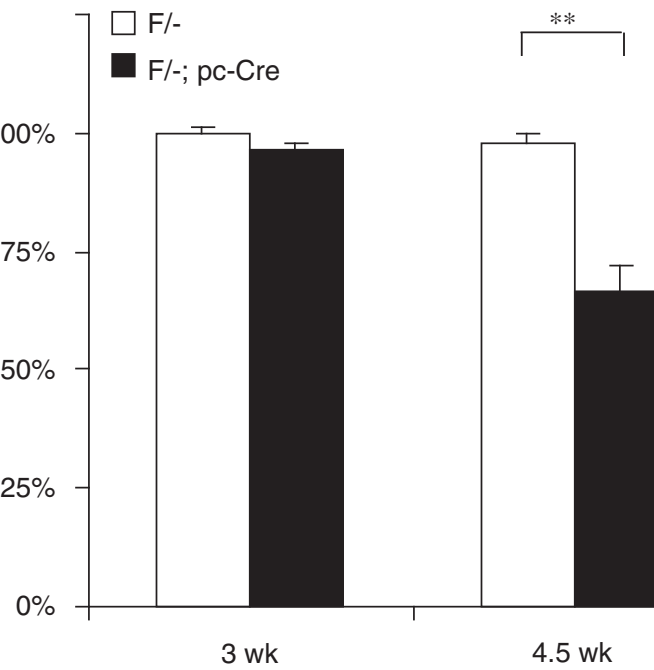

Figure 2 Grp78 Purkinje cell (PC)-specific knockout mice show accelerated PC degeneration and cerebellar atrophy. (a) Representative immunofluorescence (IF) staining for calbindin shows PC degeneration in Grp78 F/-; pc-Cre mice compared with their F/- littermates at 4.5 weeks of age. Green: calbindin, blue, DAPI. Scale bar represents $50 \mu \mathrm{m}$. (b) Representative immunohistochemical (IHC) staining for calbindin on the cerebellum sections of Grp78F/- mice and their F/-; pc-Cre littermates at 13 weeks of age. Sections were counterstained with hematoxylin. Cerebellar lobules are indicated by Roman numerals. Scale bar represents $1.5 \mathrm{~mm}$. (c) Hematoxylin and eosin (H\&E) staining on the cerebellum sections of Grp78 F/- mice and their F/-; pc-Cre littermates at indicated ages, demonstrating cerebellar atrophy. Scale bar represents $1.5 \mathrm{~mm}$. (d) Agedependent PC degeneration in Grp78 F/-; pc-Cre mice compared with their F/- littermates. The number of PCs determined by H\&E staining was quantitated within the same areas of cerebellar sections and plotted against the age of mice. The number of PCs in 3-week-old F/- mouse cerebellum was set as $100 \%$. Data are presented as mean \pm S.E.M. ${ }^{\star *} P \leqslant 0.01,{ }^{* \star *} P \leqslant 0.001$ (Student's $t$-test). For all panels, $n=3$ mice per group were examined 
Severe motor coordination defects in Grp78 PC knockout mice. Although F/-; pc-Cre mice were born with the expected Mendelian ratio and survive as well as F/F or $\mathrm{F} /$ - siblings, both male and female mice developed postnatal growth retardation when compared with $\mathrm{F} /-$ mice (Supplementary Figure S1), such that there was a significant decrease in overall body size (Figure $3 a$ ) and fasting body weight (Figure $3 b$ ). Food consumption of $\mathrm{F} /$-; pc-Cre mice was also less than that of F/- mice, which might in part account for the lower body weight (Figure 3c). By week 5.5, both male and female F/-; pc-Cre mice exhibited motor defects indicative of cerebellar ataxia. They had difficulty walking in a straight line; even while standing still the mice could not maintain balance and seemed 'tipsy' (see Supplementary Information video). The rota rod test confirmed severely impaired motor coordination ability in both male and female cohorts of F/-; pc-Cre mice, consistently over multiple trials (Figure 3d). Footprint recording further revealed an ataxic walking pattern (Figure 3e), with reduced stride/width ratio compared with $\mathrm{F} /$ - mice (Figure 3f). Interestingly, none of these phenotypes were detected in mice with PC-specific knockout of GRP94, another major ER chaperone (Supplementary Figure S2). Thus, not only that GRP78 function cannot be compensated by GRP94 but also GRP78 is uniquely required for PC survival. a

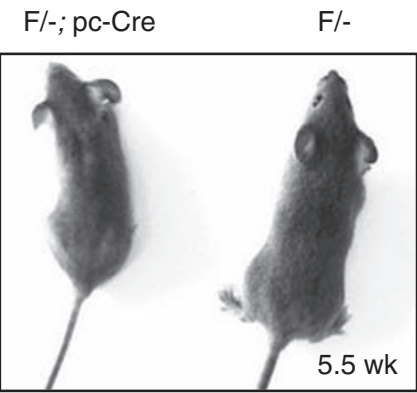

b

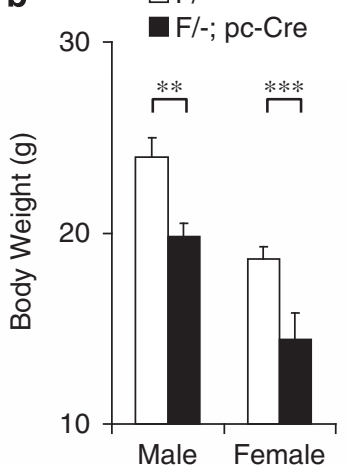

c

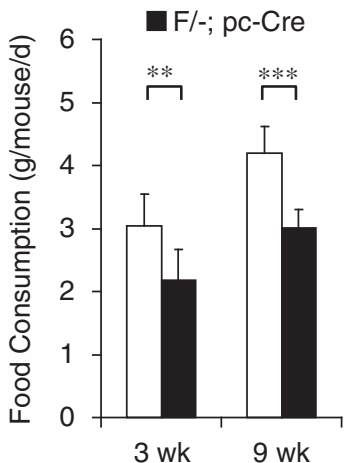

d

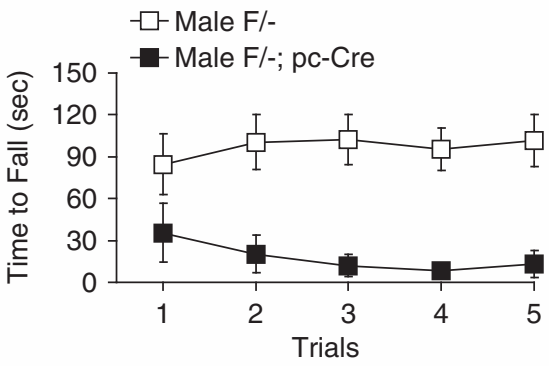

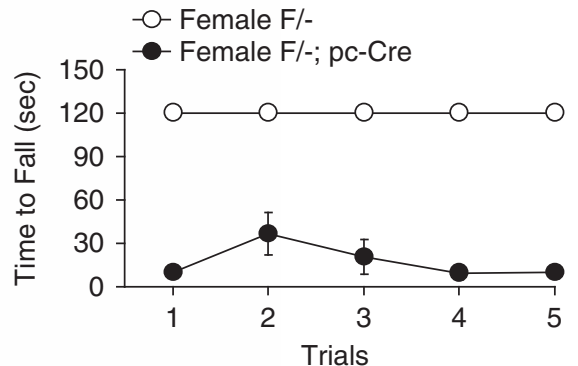

e $F /$

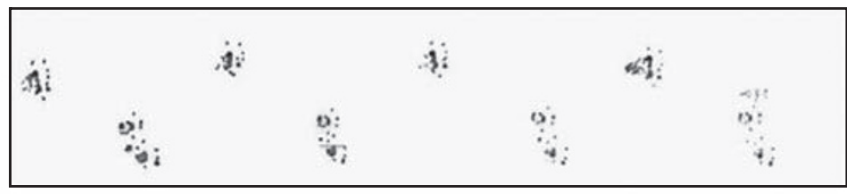

F/-; pc-Cre

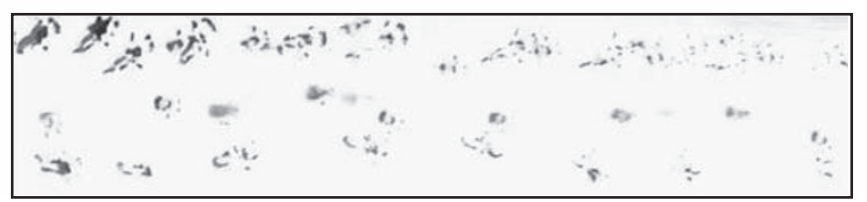

f $\square \mathrm{F} /$

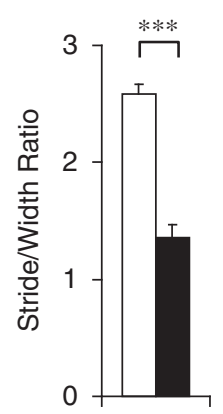

Figure 3 Behavioral abnormalities of Grp78 conditional knockout mice. (a) A representative male Grp78 F/-; pc-Cre mouse and its F/- littermate at 5.5 weeks of age. (b) Fasting body weight of 10-week-old Grp78 F/- mice and their F/-; pc-Cre littermates, both male and female; $n=10$ mice per group. (c) Food consumption of male Grp $78 \mathrm{~F} /$ - mice and their F/-; pc-Cre male littermates at 3 and 9 weeks of age. The food intakes for each individual mouse were measured for 7 consecutive days. $n=4$ mice per group. (d) Rota rod test of Grp78 F/- mice (open symbols) and their F/-; pc-Cre littermates (closed symbols) at 5.5 weeks of age. The time to fall off the rod (rotating at 30 r.p.m.) at each trial is recorded; $n=4$ to 5 mice per group. $P$-value $=0.016$ (male mice) and $P$-value $=0.008$ (female mice) (randomization test). (e) Representative footprint records of Grp78 F/- male mice and their F/-; pc-Cre male littermates at 5.5 weeks of age. The forelimbs and hindlimbs were painted blue and red, respectively. (f) The ratios of stride lengths to paw-based widths from the footprint test; $n=4$ mice per group. Data in panels $\mathbf{b}-\mathbf{d}$ and $\mathbf{f}$ are all presented as mean $\pm S$.E.M. ${ }^{* \star} P \leqslant 0.01$, ${ }^{* \star \star} P \leqslant 0.001$ (Student's $t$-test). A color version of this figure is available online 
a

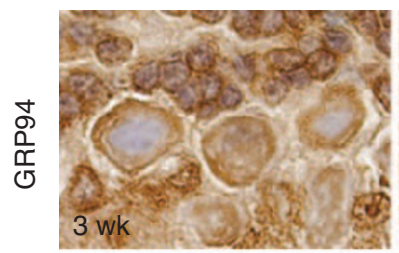

F/-; pc-Cre

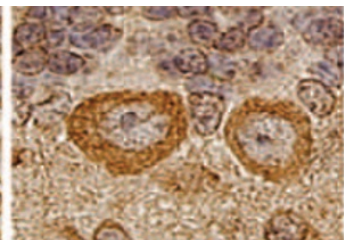

$\overline{\mathrm{Q}}$
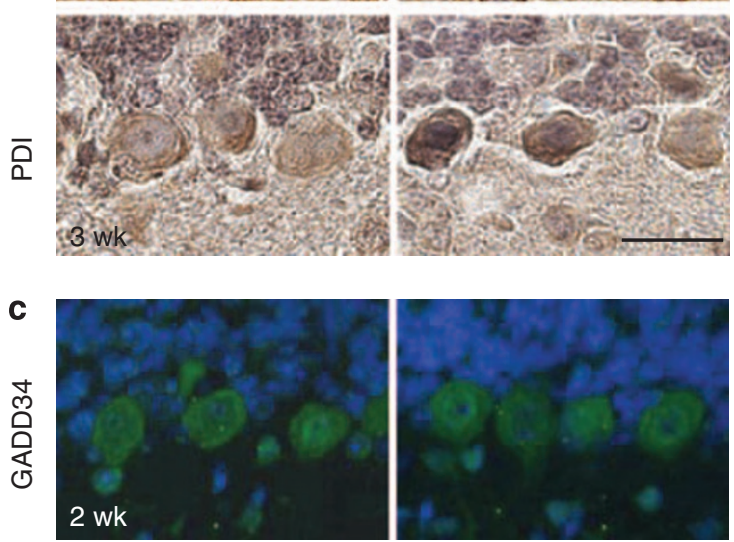

d

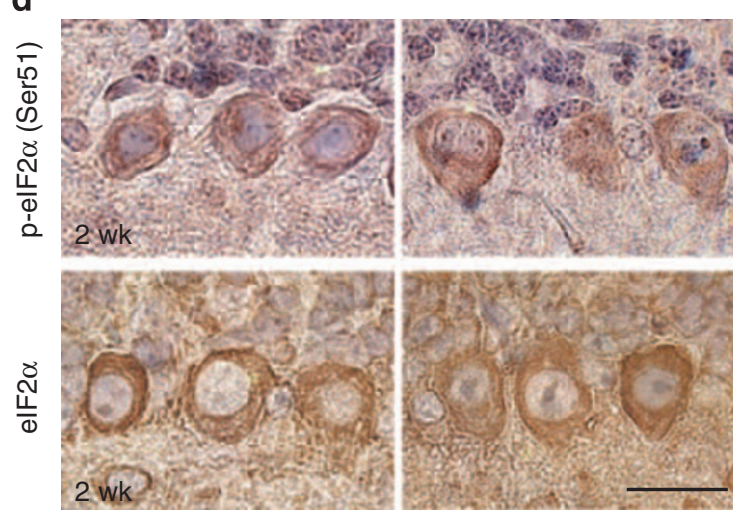

b
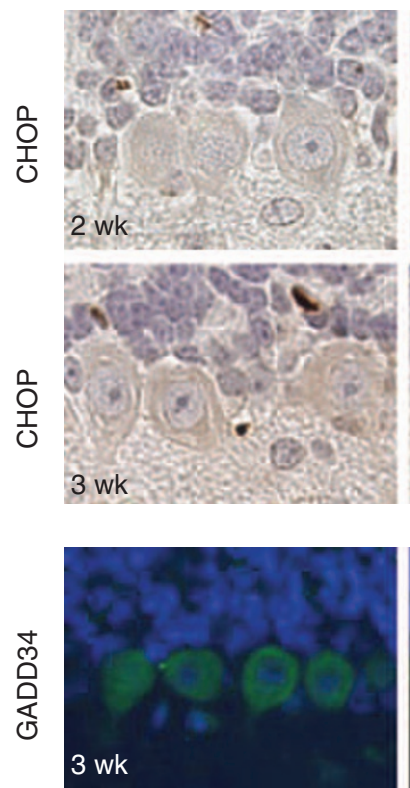

e
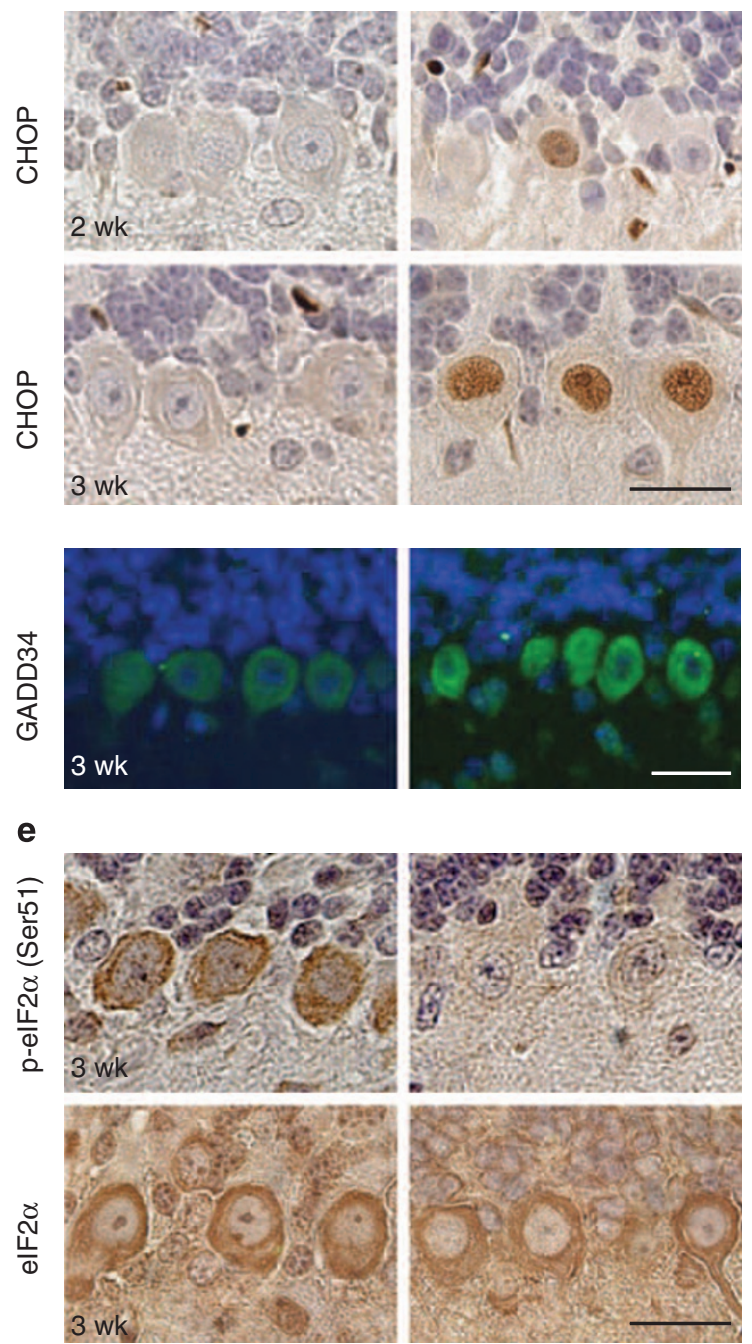

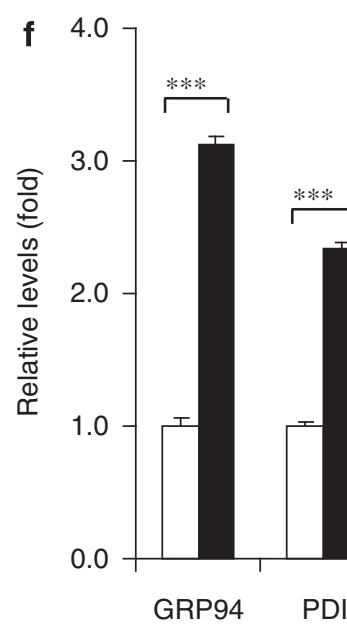

3 wk

3 wk

2 wk

3 wk

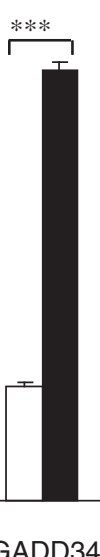

$\square \mathrm{F} /$

F/-; pc-Cre

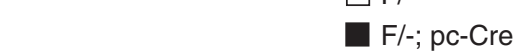


Modulation of UPR signaling pathways in GRP78-null PCs. The ability to genetically inactivate both Grp78 alleles in $P C$ s provides a unique experimental system to test directly how depletion of GRP78 affects the UPR in terminally differentiated neurons, in which ER protein quality control is central to their survival. ${ }^{20,21}$ One of the prosurvival branches of the UPR upregulates ER chaperones, such as GRP78 and GRP94, and folding enzymes, such as PDI, to alleviate protein misfolding. ${ }^{22}$ We observed that GRP94 and PDI were upregulated in almost all PCs of 3-week-old F/-; pc-Cre mice (Figure 4a). Immunostaining revealed that CHOP, an ER stress-inducible transcription factor, ${ }^{23}$ was induced in about $5 \%$ of PCs in 2-week-old F/-; pc-Cre mice; however, by 3 weeks of age, about $90 \%$ of PCs were CHOP positive (Figure 4b). ER stress activates the PERK signaling pathway, which leads to transient global translational arrest through phosphorylation of the initiation factor elF2 $\alpha$; however, this can be inhibited by a feedback mechanism mediated by GADD34, a downstream target of CHOP. ${ }^{24}$ Corresponding to CHOP induction and GADD34 upregulation in the PCs of F/-; pc-Cre mice (Figure 4c), elF2 $\alpha$ phosphorylation, which was observed at 2 weeks, was dramatically reduced by 3 weeks with the overall level of elF2 $\alpha$ unaffected (Figure $4 \mathrm{~d}$ and e). As summarized in Figure 4f, quantitation of GRP94, PDI, GADD34, phosphorylated elF2 $\alpha$ and elF2 $\alpha$ immunostaining indicates that GRP78 depletion significantly upregulates GRP94, PDI and GADD34 and suppresses elF2 $\alpha$ phosphorylation without affecting the elF2 $\alpha$ level in PCs of F/-; pc-Cre mice compared with their F/- littermates. In contrast, knockout of Grp94 did not induce UPR signaling pathways in mouse embryonic stem cells (mESCs) (Supplementary Figure S3), correlating with no neurological phenotypes in Grp94 PC-specific knockout mice. Under thapsigargin-induced ER stress, Grp94-I- and + /+ mESCs exhibited no difference in phosphorylation of PERK and elF2 $\alpha$, induction of GADD34 and splicing of XBP-1, further supporting that GRP94 did not affect UPR signaling (Supplementary Figure S3).

Reduction of cytosolic ubiquitin staining and ER expansion in GRP78-null PCs. In the mammalian ERassociated protein degradation (ERAD) mechanism, malfolded proteins released from calnexin are captured by a recognition complex containing EDEM, then passed on to GRP78 and PDI before unfolding and transport to the cytosol through the retrotranslocation mechanism, polyubiquitinated by the E1-E2-E3 system and degraded by the proteasome. ${ }^{25,26}$ For both F/- and F/-; pc-Cre mice, ubiquitin staining was detectable in both the nucleus and cytoplasm in 2-week-old cerebellar PCs (Figure 5a).
Strikingly, despite upregulation of GRP94, PDI, GADD34 and CHOP, and a readily detectable level of other cellular proteins including calbindin, elF2 $\alpha$ and $\beta$-actin (Figures 2a, 4 and data not shown), cystolic ubiquitin staining was nearly undetectable in the PCs of $\mathrm{F} /$-; pc-Cre mice by 3 weeks (Figure 5a). Quantitation of the relative ubiquitin levels shows a significantly reduced level of ubiquitin in $\mathrm{F} /-;$ pc-Cre mice at 3 weeks of age compared with their F/- littermates (Figure $5 b$ ). The immunohistochemical staining results were confirmed by immunofluorescence analysis, which also showed that, although ubiquitin staining was observed in $\mathrm{F} /$ - PCs, its level was reduced in the PCs of F/-; pc-Cre mice by week 2.5 and was barely detectable by week 3 (Figure $5 \mathrm{c}$ ). The quantitation of ubiquitin immunofluorescence indicates the gradual loss of ubiquitin level in the PCs of F/-; pc-Cre mice compared with their $\mathrm{F} /$ - littermates (Figure 5d). Reduction of ubiquitin staining was further confirmed in GRP78-null prostate epithelial cells in another Grp78 conditional knockout mouse model (Supplementary Figure S4). ${ }^{17}$ As GRP78 knockout in these cells does not affect prostate function or gross morphology, ${ }^{17}$ reduction in ubiquitin staining as a result of GRP78 depletion is unlikely the consequence of cells undergoing the cell death process.

p62/SQSTM1 is a cytosolic protein that binds to polyubiquitinated proteins and targets them to the autophagy mechanism for degradation. ${ }^{27}$ The polyubiquitinated protein aggregates stabilize $p 62 .{ }^{28}$ Consistent with the reduction in the ubiquitinated protein level, p62 level was reduced in 3-week-old GRP78-null PCs, as compared with PCs from either 2- or 3-week-old F/- mice, or 2-week-old F/-; pc-Cre mice, in which GRP78 expression was still detected (Figure 5e). Quantitation of p62 immunostaining levels in $\mathrm{F} /$ - and $\mathrm{F} /-$; pc-Cre mice showed a significant reduction by week 3 (Figure 5f). In contrast, PCs from F/- and F/-; pc-Cre mice showed similar levels of calnexin, which is an ER protein, and neurofilament and calbindin, which are cytoskeletal and cytosolic proteins, respectively (Figure 6).

Transmission electron microscopy (EM) of PCs from 3.5week-old F/-; pc-Cre mice further revealed that the ER showed prominent dilatation with accumulation of flocculant material, and in some cases, focal accumulation of electrondense material (Figure 7a and Supplementary Figure S5). No double membrane-bound inclusions were seen that would be suggestive of autophagy. ImmunoEM analysis using antibody against calnexin, an ER transmembrane protein, showed that in F/- mice, typical tubular ER structures were labeled; whereas in F/-; pc-Cre mice, expanded ER structures enclosing electron dense or flocculent materials were labeled (Figure $7 \mathrm{~b}$ ). This confirms the ER identity of the expanded

\footnotetext{
Figure 4 Modulation of UPR pathways in Purkinje cells (PCs) of Grp78 conditional knockout mice. (a, b) Representative immunohistochemical (IHC) staining for GRP94 and PDI (a) and CHOP (b) on the cerebellum sections of Grp78 F/- mice and their F/-; pc-Cre littermates at indicated ages. (c) Representative immunofluorescence (IF) staining for GADD34 on the cerebellum sections of Grp78F/- mice and their F/-; pc-Cre littermates at 2 and 3 weeks of age. Green: GADD34, blue: DAPI. (d, e) Representative IHC staining for p-elF2 $\alpha$ (Ser51) and elF2 $\alpha$ on the cerebellum sections of Grp78 F/- mice and their F/-; pc-Cre littermates at 2 and 3 weeks of age. IHC and IF staining were counterstained with hematoxylin and DAPI, respectively. All scale bars represent $25 \mu \mathrm{m}$. For panels a-e, $n=3$ mice per group were examined. (f) Quantitation of IHC and IF stainings is shown in panels a-e. The relative protein levels were quantitated by NIH software ImageJ (Bethesda, MD, USA). The mean level of staining in each group was determined by 40 randomly selected areas within PCs on the IHC or IF stained sections. The level of p-elF2 $\alpha$ was normalized to elF2 $\alpha$. For GRP94 and PDI, the staining of PCs in F/- mice at 3 weeks of age was set as 1. For GADD34, p-elF2 $\alpha$ and elF2 $\alpha$, the staining of PCs in F/- mice at 2 weeks of age was set as 1 . Data are presented as mean \pm S.E.M. ${ }^{* \star *} P \leqslant 0.001$ (Student's $t$-test)
} 
a

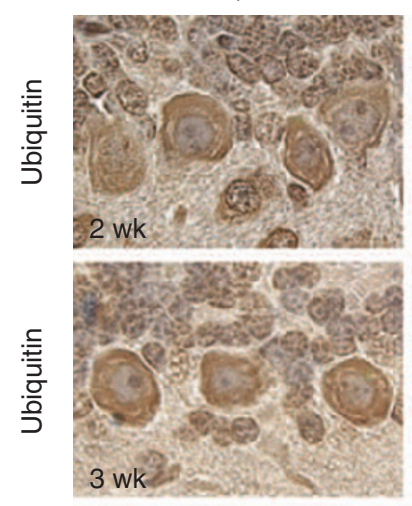

C
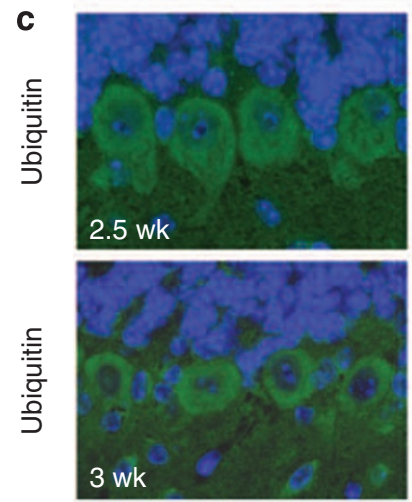

e

ֻิ

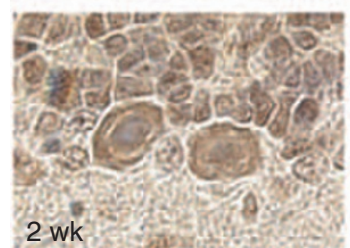

ֻัญ

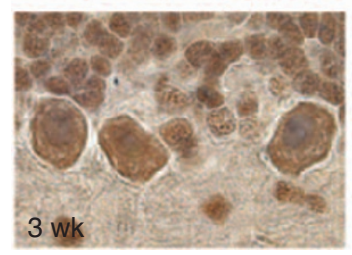

F/-; pc-Cre
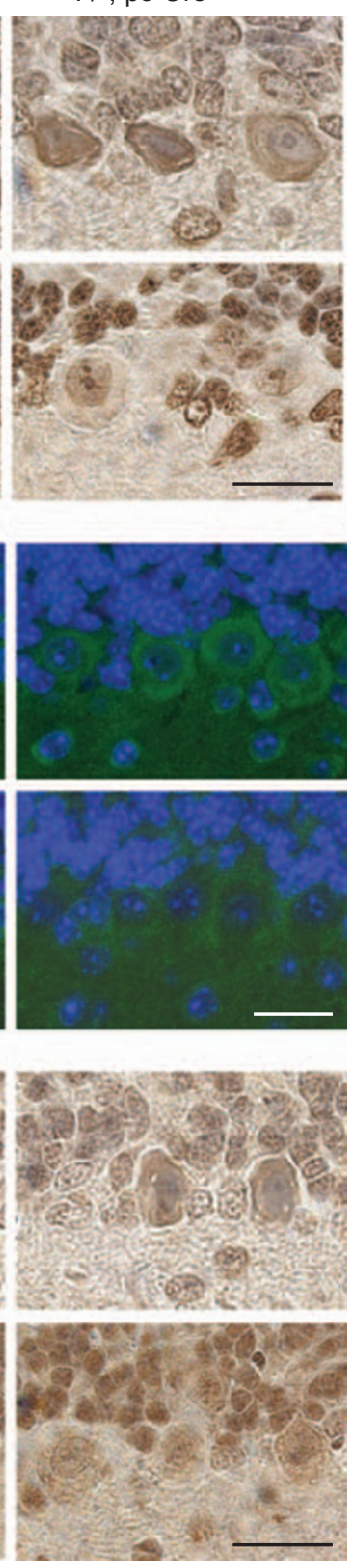

b

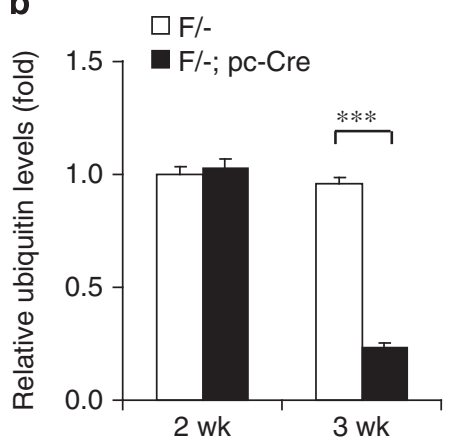

d

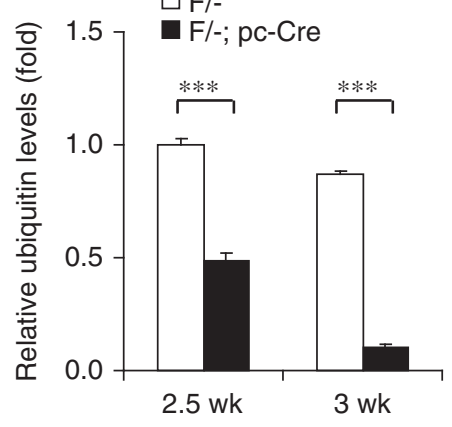

f

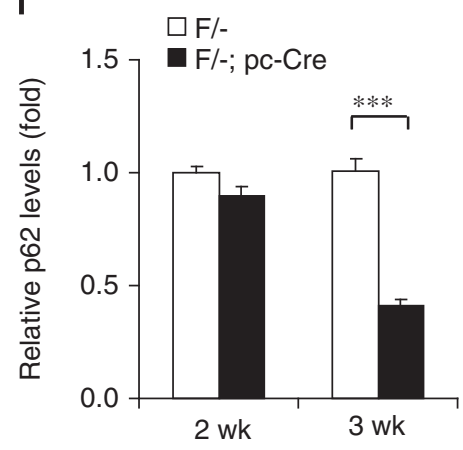

Figure 5 Reduction of protein ubiquitination and the p62 level in Purkinje cells (PCs) of Grp78 conditional knockout mice. (a) Representative immunohistochemical (IHC) staining for ubiquitin on the cerebellum sections of Grp $78 \mathrm{~F} /$ - mice and their F/-; pc-Cre littermates at 2 and 3 weeks of age. (b) Quantitation of ubiquitin immunostaining, with the level of ubiquitin staining in the PCs of F/- mice at 2 weeks of age set as 1. (c) Representative immunofluorescence (IF) staining for ubiquitin on the cerebellum sections of Grp78 F/- mice and their F/-; pc-Cre littermates at 2.5 and 3 weeks of age. (d) Quantitation of ubiquitin immunofluorescence, with the level of ubiquitin staining in the PCs of F/- mice at 2.5 weeks of age set as 1. (e) Representative IHC staining for p62 on the cerebellum sections of Grp78F/- mice and their F/-; pc-Cre littermates at 2 and 3 weeks of age. (f) Quantitation of p62 immunostaining with the level of p62 staining in the PCs of F/- mice at 2 weeks of age set as 1 . All relative levels shown in panels $\mathbf{b}$, $\mathbf{d}$ and $\mathbf{f}$ were quantitated by NIH software ImageJ. The mean level of staining in each group was determined by 40 randomly selected areas within PCs on IHC- or IF-stained sections. For all panels, $n=3$ mice per group. Data in panels $\mathbf{b}, \mathbf{d}$ and $\mathbf{f}$ are all presented as mean \pm S.E.M. ${ }^{* \star \star} P \leqslant 0.001$ (Student's $t$-test). All scale bars represent $25 \mu \mathrm{m}$

structures in PCs of F/-; pc-Cre mice. The alterations in UPR pathways and pathology observed in Grp78 F/-; pc-Cre mice are summarized in Figure 8.

\section{Discussion}

The creation of the floxed Grp78 mouse model provides a valuable tool whereby the function of GRP78, a major molecular chaperone and key rheostat for ER homeostasis, ${ }^{29}$ can be examined in the context of mammalian development and in specific adult tissues and organs. Previous studies showed that homozygous knockout of Grp78 in mouse embryos results in proliferative defect and apoptosis of the inner cell mass, resulting in lethality at E3.5. ${ }^{11}$ Nonetheless, specific knockout of Grp78 in mouse prostate epithelial cells does not affect their viability, ${ }^{17}$ and in tissue culture cell lines, 
a
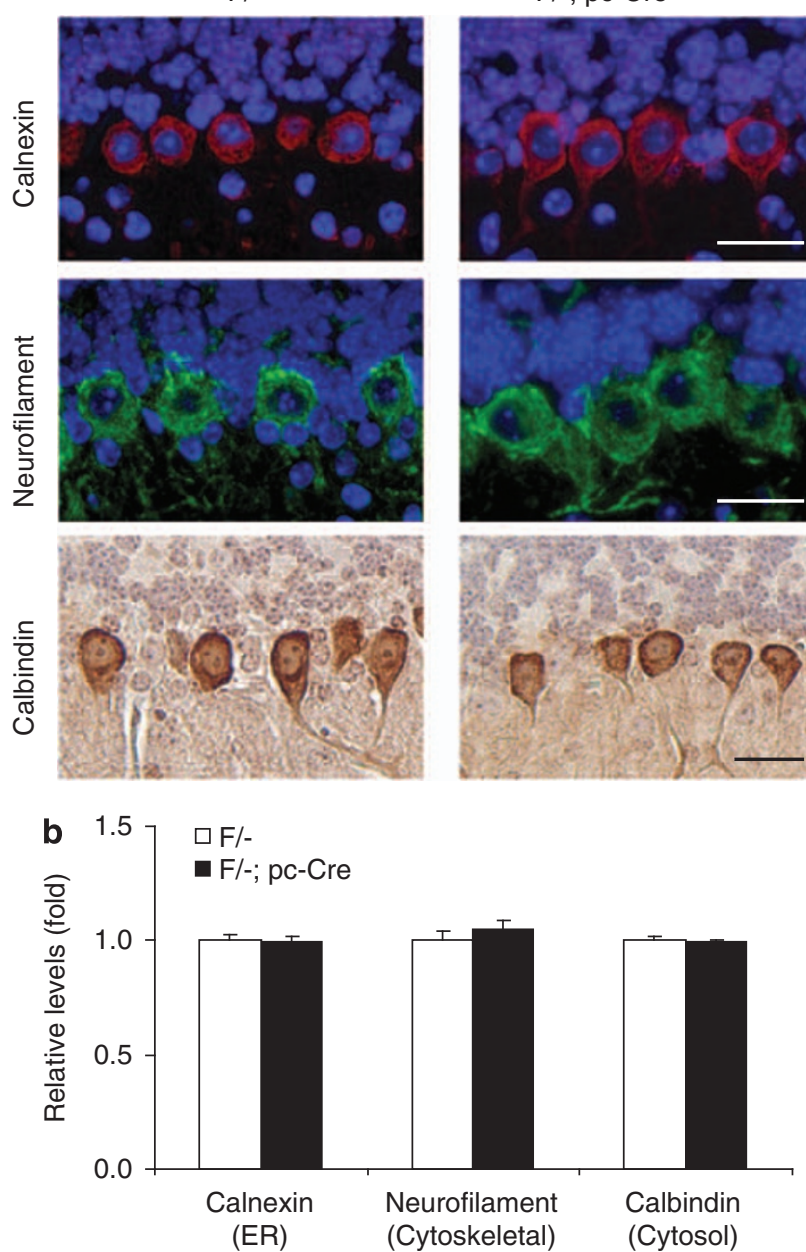

Figure 6 Similar expression levels of calnexin, nonphosphorylated neurofilament and calbindin in the Purkinje cells (PCs) of wild-type and mutant mice. (a) Representative immunofluorescence (IF) for calnexin and neurofilament and immunohistochemical $(\mathrm{IHC})$ staining for calbindin on the cerebellum sections of Grp78 F/- mice and their F/-; pc-Cre littermates at 3 weeks of age. All scale bars represent $25 \mu \mathrm{m}$. (b) Quantitation of IF and IHC stainings shown in panel a. The relative protein levels were quantitated by $\mathrm{NIH}$ software ImageJ. The mean level of staining in each group ( $n=3$ mice per group) was determined by 40 randomly selected areas within PCs on IF- or IHC-stained sections. For all quantitations, the staining of PCs in F/- mice at 3 weeks of age was set as 1. Data are presented as mean \pm S.E.M

knockdown of GRP78 by siRNA only induces apoptosis in two out of seven human cell lines being tested. ${ }^{30}$ Thus, the requirement of GRP78 in adult organs and tissues remains to be determined. Here, through the creation of the F/-; pc-Cre mouse, in which Grp78 is specifically knocked out in terminally differentiated, nonproliferating PCs, and through examination of the resulting mouse phenotypes, several novel observations are made. As evidenced in phenotypically normal F/- mice, one functional allele of Grp78 is sufficient for PC survival and cerebellum integrity, as these mice maintain normal motor function up to the age of 10 months. Interestingly, for mice over 10 months, we observed that, although female F/- mice maintain normal motor function, male F/- mice exhibit mild signs of motor deficiency on the basis of rotor rod tests (our unpublished results), suggesting an age-dependent requirement for GRP78 in maintaining cerebellum function in heterozygous males, which warrants further confirmation and investigation.

On knockout of both alleles of Grp78 in PCs, our studies revealed that mice show growth retardation, PC apoptosis, ataxia and severe defect in motor coordination by week 5 and cerebral atrophy by week 13 , directly demonstrating that GRP78 is required for survival of PCs and cerebellum integrity. Nonetheless, there are distinct differences between Grp78F/-; pc-Cre mice (referred below as the 'tipsy' mice) and SIL1-deficient 'woozy' mice, which also suffered from ER stress. A likely explanation is that SIL1 is a nucleotide exchange factor for GRP78, but is not the only one. In the GRP78 conditional knockout mouse model, PC degeneration starts at week 4.5 and all 10 lobules degenerate simultaneously and completely by 3 months, revealing that GRP78 function cannot be substituted by any other factor in all 10 lobules. In contrast, onset of PC degeneration in woozy mice starts at 3 months, and PCs in lobule $X$ and caudal lobule IX are intact even at 1 year of age and older. ${ }^{3}$ The differences in PC death kinetics and the surviving lobule pattern suggest that there are other factors that maintain GRP78 function in the PCs of woozy mice. Consistent with earlier reports that GRP170/ORP150 can serve as a nucleotide exchange factor for GRP78, ${ }^{13,14}$ it was recently discovered that brain expression of the GRP170 transgene is able to fully suppress the PC death phenotype caused by an SIL1 mutation (S Ackerman, personal communication). Furthermore, the PCs of woozy mice have highly elevated levels of GRP78 compared with wild-type control because of ER stress. Thus, despite SIL1 disruption, the residual GRP78 function in the PCs of woozy mice could mitigate the pathology and rapid loss of cerebellum function because of a complete lack of GRP78 in tipsy mice.

A previous demonstration that GRP78 depletion results in ER stress signaling was derived from ER stress titration or siRNA studies in tissue culture cells. ${ }^{10,30,31}$ The ability to completely eliminate GRP78 in PCs through genetic knockout in tipsy mice offers a unique opportunity to interrogate how a nonproliferating, terminally differentiated neuron responds to the complete loss of this UPR regulator. Although it is not possible to perform biochemical analysis as PCs constitute only a single-cell layer in the cerebellum, the large size of the $\mathrm{PC}$ with distinct nuclear and cytosolic compartments enabled us to use immunostaining techniques to determine the status of UPR that kinetically corresponds to the gradual depletion of GRP78 from these cells after activation of the cre-recombinase about 1 week after birth. We noted that it took about 2 weeks for GRP78 to be completely depleted from PCs. As a compensatory measure to GRP78 deficiency, ER chaperone GRP94 and folding enzyme PDI are upregulated, consistent with previous reports in Grp78 heterozygous mouse embryo fibroblasts ${ }^{11}$ and in established cell lines in which GRP78 was knocked down by siRNA. ${ }^{30,31}$ Also, as expected, CHOP is induced, confirming that GRP78 is a dominant suppressor of CHOP induction. ${ }^{32}$ Although the elF $2 \alpha$ level was not changed in PCs of tipsy mice, elF2 $\alpha$ phosphorylation was substantially reduced in GRP78-null PCs. One contributing factor could be the upregulation of GADD34, which is a downstream target of $\mathrm{CHOP}$ and a dominant inhibitor of elF2 $\alpha$ phosphorylation. Unfortunately, using commercially available antibodies, we 
a

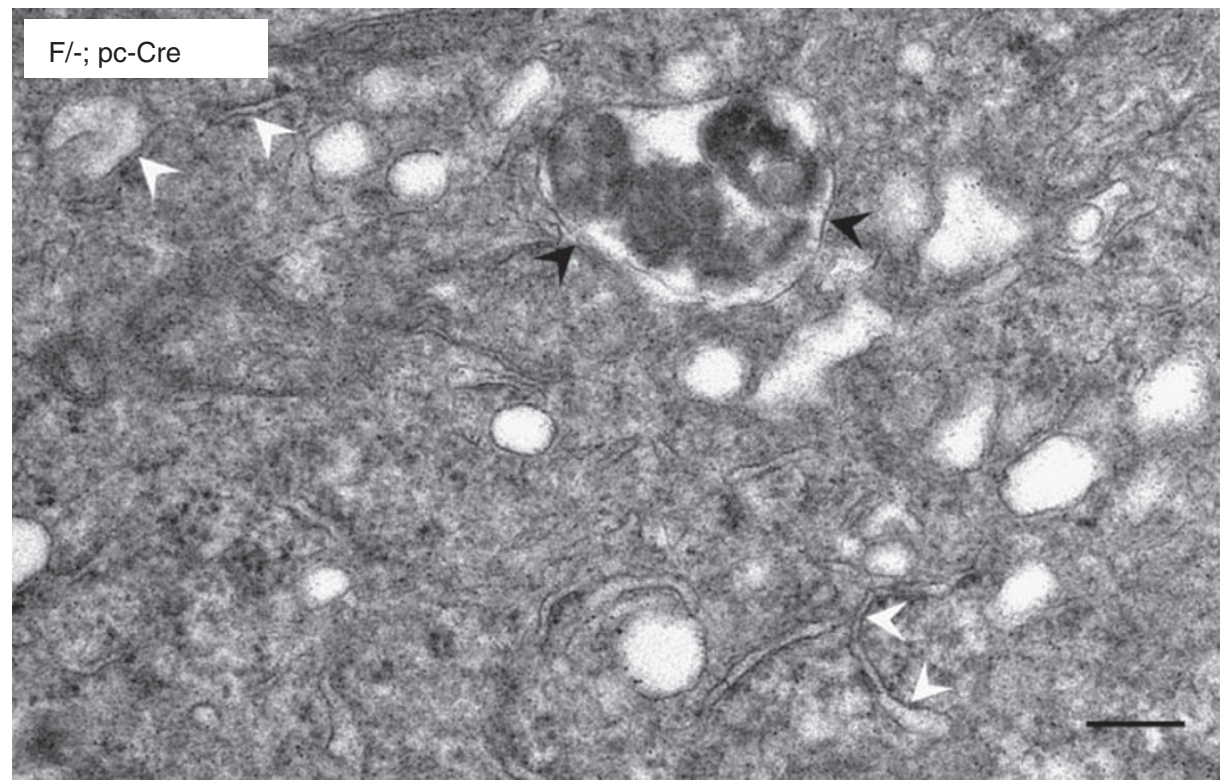

b
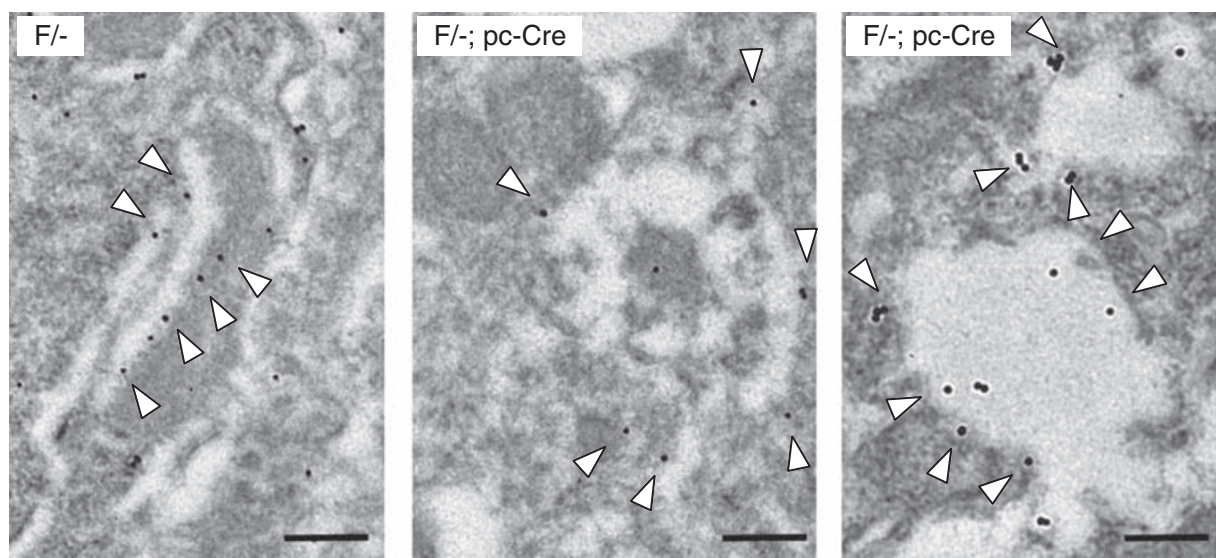

Figure 7 Altered ER structure in GRP78-null Purkinje cells (PCs). (a) Representative area of electron micrograph of a 3.5-week-old F/-; pc-Cre mouse cerebellum shows ER expansion and electron dense aggregates inside the ER in PCs. White arrowheads indicate a typical ER structure undergoing expansion. Grossly expanded ER contains diffuse accumulation of flocculent material, and in some cases, electron dense aggregates are prominent inside the ER (indicated by black arrowheads). Scale bar represents $0.5 \mu \mathrm{m}$. (b) Examples of electron micrographs showing immunogold labeling. PCs from 4.5-week-old F/- mice and their F/-; pc-Cre littermates were labeled for the ER marker calnexin. The $15 \mathrm{~nm}$ immunogold particles are indicated by open arrows. All scale bars represent $0.2 \mu \mathrm{m}$

were unable to detect in tissue sections the phosphorylation level of PERK, which is an upstream regulator of elF2 $\alpha$ phosphorylation (data not shown). Although further studies are required to determine whether alterations in the chaperone balance and UPR markers in GRP78-null PCs contribute to the pathology of Grp78 F/-; pc-Cre mice, in this study we showed that knockout of Grp94 has no effect on ER stress signaling, correlating with no PC loss and no neurological phenotype.

An unusual observation in the PCs of tipsy mice is that the level of cytosolic ubiquitin staining is dramatically reduced as GRP78 is depleted from PCs. This is not a cell-type-specific or cell death-associated observation, as this was also observed in GRP78-depleted viable prostate epithelial cells in another Grp78 conditional knockout model. ${ }^{17}$ Although we cannot exclude the possibility that PCs without GRP78 result in a dramatic decrease in the cytosolic protein substrates for ubiquitination, the same cells show robust levels of GRP94,
PDI, calnexin, GADD34, CHOP, elF2 $\alpha$, calbindin, neurofilament and $\beta$-actin. A plausible explanation is that GRP78 is required for ER protein retrotranslocation to be ubiquitinated in the cytosol. In yeast, GRP78 facilitates the export of mutant proteins to the cytosol for degradation; ${ }^{33}$ similarly, in mammalian cells, GRP78 stably binds to unfolded proteins and delivers them to the ERAD. ${ }^{34}$ The molecular mechanism of GRP78 involvement is further clarified through the recent discovery that EDEM, ERdj5 and GRP78 form a functionally important ERAD complex. ${ }^{35}$ ERdj5 functions as a disulfide reductase, which cleaves the disulfide bonds of misfolded proteins. ERdj5 binds to the ATP-bound form of GRP78 and activates ATP hydrolysis of GRP78, resulting in dissociation of GRP78 from ERdj5. In turn, the ADP-bound form of GRP78 binds protein substrates and holds them in a dislocationcompetent state until they are transferred to the retrotranslocation channel in the ER membrane for export to the 


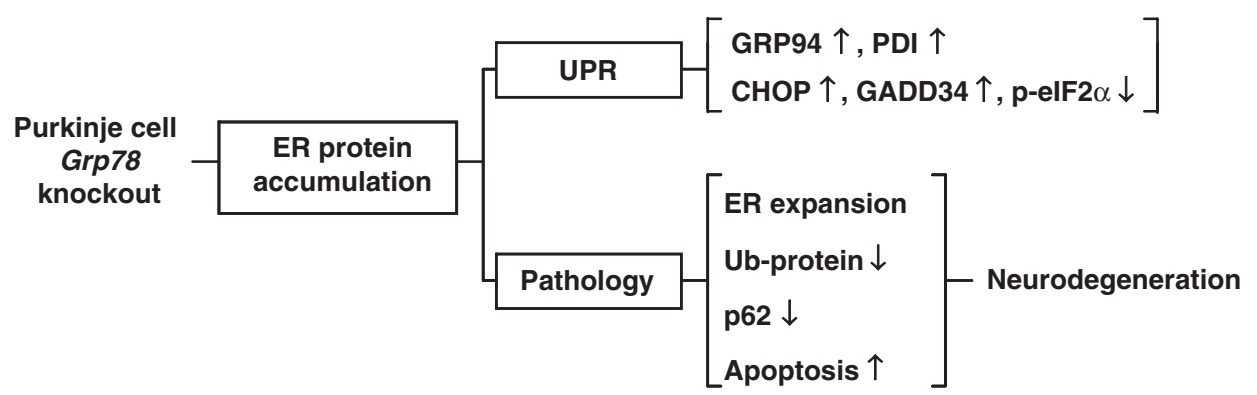

Figure 8 Summary model of modulation of the UPR and pathology resulting from Grp78 conditional knockout in PCs. GRP78 depletion in PCs leads to ER protein accumulation, abnormal ER expansion, block of protein ubiquitination and activation of the UPR and apoptosis, leading to neurodegeneration

cytosol and subsequent ubiquitination and degradation. Consistent with this model, ultrastructural images of GRP78null PCs show the accumulation of electron-dense material inside the expanded ER, but not outside the ER. Nonetheless, the tipsy mouse model does not allow us to test this mechanism biochemically. Thus, the requirement of GRP78 in protein ubiquitination remains to be determined. In addition, whether other functions of GRP78, such as binding of ER $\mathrm{Ca}^{2+}$, blocking the activation of ER-associated proapoptotic factors such as BIK and caspase- $7^{22,36,37}$ and its requirement for stress-induced autophagy, ${ }^{31}$ contribute to PC survival awaits further investigation. Besides the cerebellum, GRP78 is most likely to be important for other critical neuronal functions, as it is recently reported that knock-in mice expressing a mutant secreted form of GRP78 display disordered layer formation in the cerebral cortex and cerebellum, resulting in a neurological phenotype of reelermutant-like malformation, ${ }^{38}$ which is linked to mental disorders. ${ }^{39}$ There is also accumulating evidence for agedependent decrease in GRP78 expression or activity in specific organs and tissues, including the brain, suggesting that aging defects may result from GRP78 depletion in senescent cells. ${ }^{40}$

In summary, our studies establish Grp78 floxed mice as a valuable tool for studying the physiological role of GRP78 and the UPR in specific organs, as exemplified for the creation of tipsy mice. The latter differs from other models of $P C$ death, as only PCs, but not other neurons, are directly targeted. Considering that PCs are a part of the core circuitry in the motor learning process, tipsy mice provide a unique model for behavioral studies of PC-specific function. Furthermore, the accelerated cerebellar degeneration in these mice will facilitate the development of therapeutic agents combating ER dysfunction in neurodegeneration and other diseases. Another exciting preclinical application is that this mouse model will provide an accelerated experimental system to access stem cell transplantation for the rescue of neurological phenotypes.

\section{Materials and Methods}

Generation of PC-specific and prostate-specific Grp78 conditional knockout mice. Detailed information on the generation of Grp78 F/F, F/- mice and prostate-specific Grp78 conditional knockout mice was described previously. ${ }^{17}$ In brief, Grp78 T/ + mice ${ }^{11}$ carrying the targeted allele (T) were mated with EIIA-Cre transgenic mice, and generated offspring carrying the floxed (F) or knockout $(-)$ allele (Figure 1). PC-specific Cre (pc-Cre) transgenic mice (line L7Cre-2) were obtained from the Jackson Laboratory (Bar Harbor, ME, USA). ${ }^{18}$ Grp78 F/-; pc-Cre mice were generated through breeding between Grp78 $\mathrm{F} /$ - mice and PC-specific Cre transgenic mice. All protocols for animal use and killing were reviewed and approved by the University of Southern California Institutional Animal Care and Use Committee.

Primary antibodies. Primary antibodies used include the following: rabbit anti-GRP78 (H129) (Santa Cruz Biotechnology, Santa Cruz, CA, USA), anti-cleaved caspase-3 (Asp175) and phospho-elF2 $\alpha$ (Ser51) (Cell Signaling, Danvers, MA, USA), anti-GRP94 (SPA-851), PDI (SPA890) and calnexin (SPA-865) (Stressgen, Ann Arbor, MI, USA) and anti-p62 (SQSTM1) (BIOMOL, Plymouth Meeting, PA, USA), mouse anti-calbindin (Sigma-Aldrich, St. Louis, MO, USA), anti-CHOP (GADD153 B-3) and GADD34 (C-19) (Santa Cruz Biotechnology), anti-elF2 $\alpha$ (L57A5) and ubiquitin (P4D1) (Cell Signaling) and anti-neurofilament (nonphosphorylated) (SMI-32) (Sternberger Monoclonals, Baltimore, MD, USA).

Histological, immunohistochemical and immunofluorescence staining analyses. Mouse brains were exposed by removing the cranial bones and were fixed in situ overnight in 10\% buffered formalin. The entire brain was then removed from the skull and cut midsagittally before being embedded in paraffin using standard protocols. Immunohistochemical and immunofluorescence staining were carried out as described previously. ${ }^{19}$ In brief, the Vectastain Elite avidin-biotin complex kit (Vector Laboratories, Burlingame, CA, USA) was used for immunohistochemistry. After antigen retrieval with retrivagen $\mathrm{A}(\mathrm{pH}$ 6.0) (BD Pharmingen, San Jose, CA, USA), paraffin sections were incubated with primary antibodies in blocking solution (1.5\% serum in PBS) at $4{ }^{\circ} \mathrm{C}$ overnight with antibodies against GRP78 $(1: 100)$, calbindin $(1: 100)$, cleaved caspase-3 $(1: 50)$, GRP94 (1:200), PDI (1:300), CHOP (1:50), elF2 $\alpha$ (1:50), phospho-elF2 $\alpha$ (Ser51) $(1: 50)$, ubiquitin $(1: 200)$ and p62 $(1: 1000)$. For immunofluorescence staining, paraffin sections were incubated with primary antibodies including calbindin (1:100), GADD34 (1:200), ubiquitin $(1: 100)$ and GRP78 $(1: 100)$, calnexin $(1: 200)$ and neurofilament $(1: 100)$ at $4{ }^{\circ} \mathrm{C}$ overnight. For fluorescent detection of calbindin, GADD34, ubiquitin and neurofilament, Alexta Fluor 488conjugated goat anti-mouse IgG $(1: 200)$ (Invitrogen, Carlsbad, CA, USA) was used. For fluorescent detection of GRP78 and calnexin, Alexta Fluor 594conjugated goat anti-rabbit lgG $(1: 200)$ (Invitrogen) was used. TUNEL assay was performed using the Peroxidase In situ Apoptosis Detection Kit (Chemicon International, Billerica, MD, USA).

Motor coordination tests. The rota rod test and footprint test were performed as previously described. ${ }^{5}$ In brief, for the rota rod test, mice were placed on a rod rotating at 30 r.p.m., and the time taken for them to fall from the rod was measured. If a mouse stayed on the rod longer than the $2 \mathrm{~min}$ trial, a time of $120 \mathrm{~s}$ was recorded. For the footprint test, the forelimbs and hindlimbs of mice were painted as blue and red with nontoxic ink, respectively. Their paw placement was then recorded using a narrow tunnel $(10 \mathrm{~cm}$ wide, $40 \mathrm{~cm}$ long and $10 \mathrm{~cm}$ high) with white paper at the bottom.

Electron microscopy and immunoEM analysis. For regular EM, mouse brain tissues were dissected from the skull and immersion fixed in half strength Karnovsky's fixative (2\% paraformaldehyde and $2.5 \%$ glutaraldehyde in $0.1 \mathrm{M}$ sodium cacodylate buffer, $\mathrm{pH} 7.4$ ) for $24 \mathrm{~h}$ at $4{ }^{\circ} \mathrm{C}$. Tissues were postfixed in $1 \%$ osmium tetroxide for $2 \mathrm{~h}$ on ice. Samples were dehydrated in graded alcohol and 
infiltrated with Eponate (Ted Pella, Redding, CA). Ultrathin sections were cut at $70 \mathrm{~nm}$ and stained with uranyl acetate and lead citrate. For immunoEM labeling, mouse brain tissues were dissected from the skull and immersion fixed in fixative ( $2 \%$ paraformaldehyde and $0.1 \%$ glutaraldehyde in $0.1 \mathrm{M}$ sodium cacodylate buffer) for $24 \mathrm{~h}$ at $4^{\circ} \mathrm{C}$. Tissues were infiltrated with LR White acrylic resin (Ted Pella). After polymerization, ultrathin sections were cut at $70 \mathrm{~nm}$. After incubation with blocking solution (1\% BSA and $0.1 \%$ Triton X-100 in PBS) for 30 min, sections were stained overnight with anti-calnexin antibody $(1: 40)$ at $4{ }^{\circ} \mathrm{C}$, followed by $1 \mathrm{~h}$ incubation with $15 \mathrm{~nm}$ gold-conjugated goat anti-rabbit antibody $(1: 40)$ (Ted Pella). Sections were counterstained with uranyl acetate. All sections were examined using a JEOL JEM 2100 electron microscope (Peabody, MA) and photographed using an Orius SC1000B Gatan digital camera (Pleasanton, CA).

Body weight and food consumption measurements. Fasting body weights of the mice were measured weekly from 3 to 10 weeks of age. Food consumption was measured for seven successive days at 3 and 9 weeks of age.

Acknowledgements. We thank Dr. Robert Maxson and the Lee laboratory members for helpful discussion. We thank the University of Southern California/ Norris Comprehensive Cancer Center Transgenic Core Facility, the Cell and Tissue Imaging Core Facility, the Translational Pathology Core Facility and the Biostatistics Core Facility for assistance and consultation. This work was supported by the NIH Grants R01-CA027607, R01-CA111700 and the Freeman Cosmetic Chair to ASL.

1. Rubinsztein DC. The roles of intracellular protein-degradation pathways in neurodegeneration. Nature 2006; 443: 780-786.

2. Malhotra JD, Kaufman RJ. The endoplasmic reticulum and the unfolded protein response. Semin Cell Dev Biol 2007; 18: 716-731.

3. Zhao L, Longo-Guess C, Harris BS, Lee JW, Ackerman SL. Protein accumulation and neurodegeneration in the woozy mutant mouse is caused by disruption of SIL1, a cochaperone of BiP. Nat Genet 2005; 37: 974-979.

4. Lee JW, Beebe K, Nangle LA, Jang J, Longo-Guess CM, Cook SA et al. Editing-defective tRNA synthetase causes protein misfolding and neurodegeneration. Nature 2006; 443: $50-55$.

5. Hara T, Nakamura K, Matsui M, Yamamoto A, Nakahara Y, Suzuki-Migishima R et al. Suppression of basal autophagy in neural cells causes neurodegenerative disease in mice. Nature 2006; 441: 885-889.

6. Anttonen AK, Mahjneh I, Hamalainen RH, Lagier-Tourenne C, Kopra O, Waris L et al. The gene disrupted in Marinesco-Sjögren syndrome encodes SIL1, an HSPA5 cochaperone. Nat Genet 2005; 37: 1309-1311.

7. Senderek J, Krieger M, Stendel C, Bergmann C, Moser M, Breitbach-Faller N et al. Mutations in SIL1 cause Marinesco-Sjögren syndrome, a cerebellar ataxia with cataract and myopathy. Nat Genet 2005; 37: 1312-1314.

8. Lee AS. The glucose-regulated proteins: stress induction and clinical applications. Trends Biochem Sci 2001; 26: 504-510.

9. Hendershot LM. The ER function BiP is a master regulator of ER function. Mt Sinai J Med 2004; 71: 289-297.

10. Bertolotti A, Zhang Y, Hendershot LM, Harding HP, Ron D. Dynamic interaction of BiP and ER stress transducers in the unfolded-protein response. Nat Cell Biol 2000; 2: 326-332.

11. Luo S, Mao C, Lee B, Lee AS. GRP78/BiP is required for cell proliferation and protecting the inner cell mass from apoptosis during early mouse embryonic development. Mol Cell Biol 2006; 26: 5688-5697.

12. Sillitoe RV, Joyner AL. Morphology, molecular codes, and circuitry produce the threedimensional complexity of the cerebellum. Annu Rev Cell Dev Biol 2007; 23: 549-577.

13. Weitzmann A, Volkmer J, Zimmermann R. The nucleotide exchange factor activity of Grp170 may explain the non-lethal phenotype of loss of Sil1 function in man and mouse. FEBS Lett 2006; 580: 5237-5240.

14. Weitzmann A, Baldes C, Dudek J, Zimmermann R. The heat shock protein 70 molecular chaperone network in the pancreatic endoplasmic reticulum - a quantitative approach. FEBS J 2007; 274: 5175-5187.

15. Rutkowski DT, Kang SW, Goodman AG, Garrison JL, Taunton J, Katze MG et al. The role of p58IPK in protecting the stressed endoplasmic reticulum. Mol Biol Cell 2007; 18: $3681-3691$.
16. Petrova K, Oyadomari S, Hendershot LM, Ron D. Regulated association of misfolded endoplasmic reticulum lumenal proteins with P58/DNAJc3. EMBO J 2008; 27: 2862-2872.

17. Fu Y, Wey S, Wang M, Ye R, Liao CP, Roy-Burman $P$ et al. Pten null prostate tumorigenesis and AKT activation are blocked by targeted knockout of the stress response chaperone GRP78/BiP in prostate epithelium. Proc Natl Acad Sci USA 2008; 105: 19443-19448.

18. Barski JJ, Dethleffsen K, Meyer M. Cre recombinase expression in cerebellar Purkinje cells. Genesis 2000; 28: 93-98.

19. Dong D, Ni M, Li J, Xiong S, Ye W, Virrey JJ et al. Critical role of the stress chaperone GRP78/BiP in tumor proliferation, survival and tumor angiogenesis in transgene-induced mammary tumor development. Cancer Res 2008; 68: 498-505.

20. Rao RV, Bredesen DE. Misfolded proteins, endoplasmic reticulum stress and neurodegeneration. Curr Opin Cell Biol 2004; 16: 653-662.

21. Hetz CA, Soto $C$. Stressing out the ER: a role of the unfolded protein response in prion-related disorders. Curr Mol Med 2006; 6: 37-43.

22. Ni M, Lee AS. ER chaperones in mammalian development and human diseases. FEBS Lett 2007; 581: 3641-3651.

23. Marciniak SJ, Yun CY, Oyadomari S, Novoa I, Zhang Y, Jungreis R et al. CHOP induces death by promoting protein synthesis and oxidation in the stressed endoplasmic reticulum. Genes Dev 2004; 18: 3066-3077.

24. Marciniak SJ, Ron D. Endoplasmic reticulum stress signaling in disease. Physiol Rev2006; 86: $1133-1149$

25. Molinari M, Galli C, Piccaluga V, Pieren M, Paganetti P. Sequential assistance of molecular chaperones and transient formation of covalent complexes during protein degradation from the ER. J Cell Biol 2002; 158: 247-257.

26. Yoshida H. ER stress and diseases. FEBS J 2007; 274: 630-658.

27. Bjorkoy G, Lamark T, Brech A, Outzen H, Perander M, Overvatn A et al. p62/SQSTM1 forms protein aggregates degraded by autophagy and has a protective effect on huntingtininduced cell death. J Cell Biol 2005; 171: 603-614.

28. Seibenhener ML, Geetha T, Wooten MW. Sequestosome 1/p62-more than just a scaffold. FEBS Lett 2007; 581: 175-179.

29. Lee AS. The ER chaperone and signaling regulator GRP78/BiP as a monitor of endoplasmic reticulum stress. Methods 2005; 35: 373-381.

30. Suzuki T, Lu J, Zahed M, Kita K, Suzuki N. Reduction of GRP78 expression with siRNA activates unfolded protein response leading to apoptosis in HeLa cells. Arch Biochem Biophys 2007; 468: 1-14

31. Li J, Ni M, Lee B, Barron E, Hinton DR, Lee AS. The unfolded protein response regulator GRP78/BiP is required for endoplasmic reticulum integrity and stress-induced autophagy in mammalian cells. Cell Death Differ 2008; 15: 1460-1471.

32. Pyrko P, Schonthal AH, Hofman FM, Chen TC, Lee AS. The unfolded protein response regulator GRP78/BiP as a novel target for increasing chemosensitivity in malignant gliomas. Cancer Res 2007; 67: 9809-9816.

33. Plemper RK, Bohmler S, Bordallo J, Sommer T, Wolf DH. Mutant analysis links the translocon and BiP to retrograde protein transport for ER degradation. Nature 1997; 388 . 891-895.

34. Skowronek MH, Hendershot LM, Haas IG. The variable domain of nonassembled Ig light chains determines both their half-life and binding to the chaperone BiP. Proc Natl Acad Sci USA 1998; 95: 1574-1578.

35. Ushioda R, Hoseki J, Araki K, Jansen G, Thomas DY, Nagata K. ERdj5 is required as a disulfide reductase for degradation of misfolded proteins in the ER. Science 2008; 321: 569-572.

36. Reddy RK, Mao C, Baumeister P, Austin RC, Kaufman RJ, Lee AS. Endoplasmic reticulum chaperone protein GRP78 protects cells from apoptosis induced by topoisomerase inhibitors: role of ATP binding site in suppression of caspase-7 activation. $J$ Biol Chem 2003; 278: 20915-20924.

37. Fu Y, Li J, Lee AS. GRP78/BiP inhibits endoplasmic reticulum BIK and protects human breast cancer cells against estrogen-starvation induced apoptosis. Cancer Res 2007; 67: 3734-3740.

38. Mimura N, Yuasa S, Soma M, Jin H, Kimura K, Goto S et al. Altered quality control in the endoplasmic reticulum causes cortical dysplasia in knock-in mice expressing a mutant $\mathrm{BiP}$. Mol Cell Biol 2008; 28: 293-301.

39. Impagnatiello F, Guidotti AR, Pesold C, Dwivedi Y, Caruncho H, Pisu MG et al. A decrease of reelin expression as a putative vulnerability factor in schizophrenia. Proc Natl Acad Sci USA 1998; 95: 15718-15723.

40. Naidoo N. ER and aging-Protein folding and the ER stress response. Ageing Res Rev 2009; 8: 150-159.

\section{Supplementary Information accompanies the paper on Cell Death and Differentiation website (http://www.nature.com/cdd)}

